\title{
Nano-enhanced optical gene delivery to retinal degenerated mice
}

\author{
Subrata Batabyal , Sivakumar Gajjeraman, Sulagna Bhattacharya, Weldon Wright, Samarendra Mohanty*
}

Nanoscope Technologies LLC, 1312 Brown Trail

Bedford, Texas, USA, 76022

†These authors contributed equally.

Corresponding Author

* E-mail: smohanty@nanoscopetech.com 


\begin{abstract}
The efficient and targeted delivery of genes and other impermeable therapeutic molecules into retinal cells is of immense importance for therapy of various visual disorders. Traditional methods for gene delivery require viral transfection, or chemical methods that suffer from one or many drawbacks such as invasiveness, low efficiency, lack of spatially targeted delivery, and can generally have deleterious effects such as unexpected inflammatory responses and immunological reactions. Here, we introduce a continuous wave near-infrared laser-based Nano-enhanced Optical Delivery (NOD) method for spatially controlled delivery of opsin-encoding genes into retina in-vivo. In this method, the optical field enhancement by gold nanorods is utilized to transiently permeabilize cell membrane enabling delivery of exogenous impermeable molecules to nanorod-binding cells in laser-irradiated regions. The successful delivery and expression of opsin in targeted retina after in-vivo NOD in the mice models of retinal degeneration opens new vista for re-photosensitizing retina with geographic atrophies as in dry age-related macular degeneration (AMD).
\end{abstract}

KEYWORDS: Ocular gene therapy, optical delivery, optogenetics, dry-AMD. 


\section{Introduction}

Dry age-related macular degeneration (Dry-AMD) involving dysfunction of retina is characterized by degeneration of photoreceptors. The degree of visual loss increases with ageing and this is a major concern for our demographic changes towards elderly population. The eye offers an excellent target for gene therapy (replacement of defective genes with new functional genes) because it is easily accessible and relatively immune privileged. Most of the current clinical ophthalmic treatments using gene', RNA aptamer $^{2}$ and shRNAs ${ }^{3}$ for therapeutic benefits are utilizing viral vectors, which suffers from drawbacks such as low efficiency, unexpected inflammatory responses, especially innate and immune barriers ${ }^{4}$, toxicity $^{5}$, and potential recombination of or complementation in vivo which can generate virulent viral pathogens after vector delivery ${ }^{6}$. In addition, limitation on size of plasmid that can be packaged by the mostcommonly used safe (AAV) virus vectors ${ }^{7.8}$ may create hindrance to deliver novel gene-editing large molecules such as CRISPR/Cas9 for visual disorders. Furthermore, the ability to deliver therapeutic genes into spatially targeted higher order retinal neurons of degenerated retina (e.g. geographic atrophic areas of macula in AMD ${ }^{913}$ ) is advantageous, for example in optogenetic activation therapy. Therefore, there have been significant efforts in developing alternative, non-viral methods such as physical (electroporation $^{14.15}$, ultrasound ${ }^{16}$ etc.) and chemically-mediated (e.g. biodegradable polymer ${ }^{17,18}$ ) methods ${ }^{1920}$. These methods also suffer from one or more drawbacks, such as invasiveness, reduced efficiency, lack of spatially-targeted delivery and/or having several deleterious effects on transfected cells ${ }^{21}$. The advent of optoporation by focused ultrafast near infrared laser microbeam has allowed mitigating the drawbacks of these methods. Recently, we demonstrated use of near IR laser microbeam for delivery of impermeable molecules to neurons ${ }^{22}$, opsin-encoding genes to cells ${ }^{23}$ and localized areas of the retina ${ }^{24}$. However, the extreme difficulties in maintaining tight focus inside tissue and requirement of high peak power to create holes in the cell membrane, has limited the use of laser-based delivery to small animal applications $^{25}$. Therefore, there is an imminent need for development and optimization of new and efficient non-viral method that can deliver therapeutic molecules irrespective of their size to spatially targeted regions of retina in a minimally invasive manner.

Recently, optogenetic therapies have been evaluated for vision restoration in mice models of retinal degeneration either by non-specific stimulation of retina ${ }^{26}$ or in a cell-specific manner for retinal ganglion cells $\mathrm{s}^{27.31}$ and $\mathrm{ON}$ bipolar cells $\mathrm{s}^{323334}$. Further, use of chloride-channel opsin (NpHR) expressing in longerpersisting cone photoreceptor protein has shown promise for restoration of vision ${ }^{35}$. The primary method for delivery of opsin-encoding genes to adult retina employs viral transfection which has high transduction efficiency and persistent gene expression ${ }^{36}$. Both non-specific transfection of the retina as well as 
promoter-specific targeting of retinal ganglion cells of adult mice and rats using viral methods have been successful ${ }^{27303,3233,37-38}$. However, clinical translation of optogenetic activation for vision restoration has a major challenge: (i) requirement of external activation light or opsin with significantly higher sensitivity to ambient light; and (ii) method to optogenetically treat only those areas of the retina that have undergone degeneration rather than the whole retina. Effective therapy of visual disorders involving geographic atrophies of the retina requires localized delivery of the targeted molecules to specific retinal cells in atrophied regions.

Herein, we introduce use of nano-enhancement of near-infrared optical field of continuous wave (CW) near-infrared (NIR) laser beam by surface plasmon resonance (SPR) near targeted retinal cells for gene delivery. To allow ambient-light based activation ${ }^{34}$, we utilized multi-characteristic-opsin (MCO) encoding plasmids to sensitize retinal cells in degenerated retina. It would be highly desirable to reinduce transgene expression by reinjection, and NOD provides this opportunity.

\section{Materials and Methods}

Ethics statement

All experimental procedures were conducted according to the Nanoscope Technologies-Institutional Animal Care and Use Committee approved protocol.

\subsection{Construction, and Purity of plasmid}

Double stranded DNA fragment coding for multi characteristic opsin-II (MCO-II) was synthesized using high throughout DNA synthesizer. The restriction enzyme sites (BamH1 and Sal1) were added to the ends of the DNA fragment by PCR amplifying using the corresponding primers. Afterwards, the fragment with BamH1/Sal1 sites was inserted to the BamH1/Sal1 site of the pAAV vector. Thus, a plasmid was created comprising a CAG promoter, MCO-II and reporter (mCherry). Prior to the experiments, the plasmid was digested by restriction enzymes (BamH1 and Sal1) to verify the size and purity using $0.8 \%$ agarose gel electrophoresis.

\subsection{Theoretical structure prediction}

Raptor-X used for predicting the 3-D structure of MCO-II. The program uses a conditional neural fields (CNF), a variant of conditional random fields and multiple template treating procedure to develop the predicted structure of MCO-II gene. 
2.3 Sensitizing/functionalizing gold nanorods to optical delivery beam and cells

The aspect ratio (ratio of size of short axis to long axis) of gold nanorods (GNRs) can vary from 1:1 to $1: 5$ to result in Surface plasmon resonance (SPR) peak varying from visible $(\sim 530 \mathrm{~nm})$ to NIR $(\sim 1100$ $\mathrm{nm}$ ). Gold nanorods (diameter: $10 \mathrm{~nm}$, length: $40 \mathrm{~nm}$ ) with plasmon absorption maximum at $800 \mathrm{~nm}$ were used for NOD experiments. For specific membrane binding, gold nanorods were incubated with $4 \mu \mathrm{M}$ Concanavalin $\mathrm{A}$ at $37{ }^{\circ} \mathrm{C}$ (Overnight). The unbound ConA molecules were removed by centrifugation at 13,000 rpm for $10 \mathrm{~min}$. The ConA conjugated GNR were then resuspended into 10mM HEPES buffer pH (7.2). Gold nanorods (diameter: $10 \mathrm{~nm}$, length: $40 \mathrm{~nm}$ ) with SPR maximum at $808 \mathrm{~nm}$ was used for NOD optimization.

\subsection{Patch-clamp recording setup}

The MCO-II opsin is activatable over a broad visible-light spectral range (400 to 700nm). To determine the light dependent inward photocurrent, the MCO-II expressing cells were exposed to pulses (500 ms) of light with varying intensities. A single mode optical fiber coupled to a supercontinuum laser source (NKT Photonics) delivered the broadband light to the sample for optogenetic stimulation. A power meter (818-SL, Newport) was used to quantify the light intensity at the sample plane. The light pulse width was synchronized with the electrophysiology recording system, controlled by Axon Instruments Digidata system (Molecular Devices). Cells, transfected with CAG-MCO-II-mCherry were incubated with all-trans retinal (ATR, $1 \mu \mathrm{M}$ ) for 4 hours before conducting the patch clamp experiments.

The patch-clamp recording setup consists of an inverted Nikon fluorescence microscope (TS 100) platform using an amplifier system (Axon Multiclamp 700B, Molecular Devices). Micropipettes were pulled using a two-stage pipette puller (Narshinghe) to attain resistance of 3 to $5 \mathrm{M} \Omega$ when filled with a solution containing (in $\mathrm{mM}$ ) $130 \mathrm{~K}$-Gluoconate, $7 \mathrm{KCl}, 2 \mathrm{NaCl}, 1 \mathrm{MgCl}_{2}, 0.4$ EGTA, $10 \mathrm{HEPES}$, 2 ATP-Mg, 0.3 GTP-Tris and 20 sucrose. The micropipette-electrode was mounted on a micromanipulator. The extracellular solution contained (in $\mathrm{mM}$ ): $150 \mathrm{NaCl}, 10$ Glucose, $5 \mathrm{KCl}, 2 \mathrm{CaCl}_{2}, 1 \mathrm{MgCl}_{2}$ was buffered with $10 \mathrm{mM}$ HEPES (pH 7.3). Photocurrents were measured while holding cells in voltage clamp at $-70 \mathrm{mV}$. The electrophysiological signals from the amplifier were digitized using Digidata 1440 (Molecular devices), interfaced with patch-clamp software (Clampex, Molecular Devices). pClamp 10 software was used for data analysis. 
2.5 Delivery and expression of MCO-II- mCherry using NOD: HEK cells and retinal explants of rd10 mice

The HEK-293 cells were grown in static culture and maintained in Dulbecco's modified Eagle's medium (DMEM)/F-12 with 10\% fetal bovine serum, $0.2 \mathrm{mg} / \mathrm{mL}$ streptomycin, and $200 \mathrm{U} \mathrm{mL}$ penicillin. The cultures were maintained at $37{ }^{\circ} \mathrm{C}$ in a humidified $5 \% \mathrm{CO}_{2}$ atmosphere. Prior to the NOD experiments, the cells were incubated with ConA functionalized GNR for two hours at $37^{\circ} \mathrm{C}$ in a humidified $5 \% \mathrm{CO}_{2}$ incubator. Purified plasmid (CAG-MCO-II-mCherry) was added in the cell culture medium and incubated for $15 \mathrm{~min}$ at $37{ }^{\circ} \mathrm{C}$ in a humidified $5 \% \mathrm{CO}_{2}$ incubator. After 15 minutes of incubation, NOD was carried out using CW laser (800 nm).

\subsection{Animal preparation}

B6.CXB1-Pde6brio/J mice (Jackson Laboratory, Bar Harbor, ME) were used in this study. The mice were housed in the Nanoscope technologies animal facility. All animal experimentation was conducted under local IACUC approved protocols in accordance with the ARVO statement for the Use of Animals in Ophthalmic and Vision Research. Mice were maintained on a 12:12 light cycle (lights on at 07:00). At least 6 eyes were used for in-vitro and in-vivo NOD experiments. Retinal degenerated mice ( $r d 10$ : B6.CXB1-Pde6b $\left.\mathrm{b}^{\mathrm{r} 10} / \mathrm{J}\right)$ were obtained from Jackson laboratory and bred in the animal facilities of the Nanoscope. Once the animals were acclimated to the animal facility for $\sim 1$ week, they were anesthetized with $90 \mathrm{mg} / \mathrm{kg}$ ketamine, $10 \mathrm{mg} / \mathrm{kg}$ xylazine acepromazine $(0.5 \mathrm{mg} / \mathrm{kg})$. In all experiments attention was paid to the ethical guidelines for investigations of experimental pain in conscious animals, and the procedures were approved by the IACUC.

\subsection{Retinal explant}

Adult $r d 10$ deficient mice were sacrificed using $\mathrm{CO}_{2}$. The eyes of $r d 10$ mice were surgically removed from its muscles and remaining orbital contents and then choroid, sclera, cornea, lens, vitreous were the removed from enucleated eye. The remaining retina was incubated with fresh Neurobasal media. The RPE was gently removed from retina and retinal explants then cut into pieces using a tissue chopper. With the photoreceptor side down, the explants were placed onto sterilized petri-dishes previously coated with 5ug/dish of Poly-D-Lysine containing $1 \mathrm{ml}$ of Neurobasal media. The explants were incubated with ConA conjugated GNR for $2 \mathrm{hr}$ at $37{ }^{\circ} \mathrm{C}$ in a humidified $5 \% \mathrm{CO}_{2}$ incubator. The CAG-MCO-IImCherry plasmid was placed directly on the retinal surface for $15 \mathrm{~min}$ and explants were then exposed with $800 \mathrm{~nm}$ CW laser for $30 \mathrm{sec}$, the GNRs are functionalized with ConA. 


\subsection{Transduction of MCO-II-mCherry into $r d 10$ mouse retina}

Adult (8 weeks old) $r d 10$ mice were treated humanely in strict compliance with IACUC on the use of animals in research. The $\mathrm{rd10}$ mice $(\mathrm{N}=3)$ were anesthetized with a mixture of ketamine $(65 \mathrm{mg} / \mathrm{kg})$, xylazine $(7.5 \mathrm{mg} / \mathrm{kg})$, and acepromazine $(0.5 \mathrm{mg} / \mathrm{kg})$. One drop of local anesthesia (0.5\% proparacaine hydrochloride) was instilled into both the eyes of the animals. Functionalized gold nanorods and MCOII-mCherry plasmids was injected into one of the eyes by a sterilized 32-G needle of a Hamilton microsyringe inserted through the sclera into the vitreous cavity. As a negative control, the other eye was intravitreally injected with same volume of PBS. 1\% Tropicamide ophthalmic solution was applied for dilating the pupil. The cornea was kept moist with a balanced salt solution during the entire surgical procedure. Schematic of experimental procedure for in-vivo NOD is shown in Fig. 4a. NOD using CW laser was carried out with the in-vivo set up (Fig. 4b). After 1 week of transfection, the animals were euthanized, and retinal tissue explanted. Using confocal microscope (Fluoview FV1000) for mCherry expression, the expression level (fluorescence intensity) of these cells were quantified. Image processing was performed by using NIH ImageJ software.

\subsection{Fourier domain optical coherence tomography system}

FD-OCT is based on spectral domain implementation of the OCT system developed in the OCT research community ${ }^{39.4}$, and has been in clinical Ophthalmology practice. In the FD-OCT, reference mirror is not scanned to perform depth scan. The reference mirror is stationary. The interference signal between the reflected intensities from the reference mirror and the sample microstructures is detected with a spectrometer as a function of wavelength. The detected signal (as a function of wavelength) is then Fourier transformed to obtain intensity profile as a function of depth. FD-OCT scans the whole depth of the sample without any mechanical scanning. We performed SD-OCT imaging as described previously ${ }^{4547}$. Prior to imaging, mice were anesthetized. Mice were placed on the platform of the SD-OCT and retinal thickness measurements were made.

\subsection{Statistics}

GraphPad Prism was used to analyze data. The data were plotted as mean \pm S. D. Statistical significant difference analyses were carried out by t-test. $P<0.05$ was considered statistically significant.

\section{Results}

3.1. Improvement of optokinetic response in MCO-II transfected rd10 
The rd10 mice with MCO-transfected retina demonstrated increase in optokinetic response at different speed of the vertical stripes $(0.1 \mathrm{cpd})$ at ambient light level. The basic principle of this assay is: whenever a moving pattern is presented to an animal, the (light sensing) animal will move its head as a transient corrective measure to maintain stable vision. The advantage of this method is that it does not require any previous training of the animal. Quantitative comparison of number of head movement before and after (2, 4 weeks) MCO-II transfection is shown in Suppl. Fig. 1. Quantitative comparison of number of head movement at different speed of rotation of the vertical stripes: (a) $2 \mathrm{rpm}$ and (b) $8 \mathrm{rpm}$ before and after MCO-II transfection. rd10 mice with MCO-II transfection shows improved optokinetic response as reflected in the increase head movement. The average light intensity at the center of the chamber was $0.001 \mathrm{~mW} / \mathrm{mm}^{2}$.

\subsection{Nano-enhanced Optical Delivery: Principle and in-vitro optimization}

The principle of Nano-enhanced Optical Delivery (NOD) is shown in Fig. 1a and 1b. The ConA conjugated gold nanorods (injected intravitreally) bind to the membrane of the targeted retinal cells. Upon illumination of a continuous wave laser of wavelength $(800 \mathrm{~nm})$, the enhanced photothermal properties of gold nanorods on the cell membranes resulted in localized temperature increase allowing the delivery of impermeable molecules via cell membrane. Fig. 1c shows electron microscopic image of gold nanorods used for optical enhancement of laser beam at the ends of the rods. The measured optical density of gold nano-rods with peak at $800 \mathrm{~nm}$ is shown in Fig.1d.

NOD was used for spatially targeted delivery of ambient-light activatable multi-characteristic opsin (MCO-II) into mammalian cells. A typical circular map showing the insertion of MCO-II gene cloned in pAAV vector at the restriction sites: EcoR1 and Sal1 is depicted in Fig. 2a. The size of the vector and DNA fragment (MCO-II-mCherry) was further verified using the restriction enzymes as shown in the Fig. 2b. Fig. 2c illustrates the three-dimensional arrangement of amino acid chains of MCO-II obtained by theoretical modeling. The secondary structure prediction revealed that MCO-II opsin contains $46 \%$ of alpha helix, 17\% beta sheet and 36\% random coil structure. For optimization of NOD-based gene delivery, in-vitro experiments were performed for delivery of plasmids encoding for MCO-II-mCherry into HEK-293 cells. The cells were exposed for different durations to CW near-infrared laser beam with intensity of $6.4 \mathrm{~mW} / \mathrm{mm}^{2}$. Fig. 2d shows a representative confocal microscopic image of HEK cells 2 days after NOD of MCO-II-mCherry plasmids in the rectangular area using 1 min exposure of CW nearinfrared laser beam. The NOD-based gene delivery efficiency at different laser exposures was quantified based on the measured mCherry (reporter) fluorescence intensity of cells (averaged) in targeted areas. 
Fig. 2e shows the variation of mCherry fluorescence as function of NOD laser beam exposure time varying from 1 to $4 \mathrm{~min}$.

To determine functioning of the MCO-II expressing cells, patch-clamp electrophysiology was carried out in the presence of white light stimulation. The representative inward current in MCO-II expressing cells in response to light $\left(0.024 \mathrm{~mW} / \mathrm{mm}^{2}\right)$ is shown in Fig. $2 \mathrm{f}$. The peak photocurrent generated in MCO-II expressing cells was found to be $300 \mathrm{pA}$. While the on-rate of induced photocurrent in MCO-II expressing cells in response to the light was in ms range, the off rate (decay of current in absence of light) was found to be significantly higher.

\subsection{Nano-enhanced optical delivery of opsin-plasmids into retina explant}

We utilized NOD parameters optimized (in HEK cells) for spatially targeted delivery of MCO-IImCherry plasmids into retina explants. The explants were incubated for $1 \mathrm{hr}$ with ConA-conjugated GNRs having peak absorption at $800 \mathrm{~nm}$. Confocal fluorescence image of retinal explant (Fig. 3a) shows reporter-mCherry expression in targeted area (marked by square) 2 days after exposure to CW NIR laser beam $(800 \mathrm{~nm})$. Fig. 3b shows cellular expression in the targeted area of the explant. The nontargeted areas (Fig. 3c) showed basal level of autofluorescence. To quantify the relative expression of mCherry in cell membrane and intracellular components, intensity profiles along line drawn across the cells are plotted (Fig. 3d). The MCO-II expression in plasma membrane was found to be significantly higher than intracellular expression. Comparison of MCO-II-mCherry expression (measured by average mCherry fluorescence intensity) in targeted versus non-targeted regions in retina explant is shown in Fig. 3e. No detectable increase in fluorescence in mCherry band was observed in non-laser exposed explants incubated with GNR and MCO-II-mCherry plasmids. Further, the explants irradiated with CW NIR laser beam and MCO-II-mCherry plasmids only (and no GNRs) did not exhibit any rise in cellular fluorescence in mCherry band.

\subsection{In-vivo targeted delivery of MCO-genes to retina using NOD}

After successful in-vitro delivery of MCO-II plasmids to retinal explants, nano-enhanced optical delivery experiments were carried out in-vivo using the CW NIR laser beam (800 nm) to achieve spatially targeted gene expression in the retina of $r d 10$ mice with photoreceptor degeneration. The experimental steps for in-vivo NOD into retina are shown in Fig. 4a. Fig. 4b shows the schematic of the experimental NOD setup. The eye was exposed with the CW NIR laser beam with optimized parameters (intensity: 20 
$\mathrm{mW} / \mathrm{mm}^{2}$, exposure time: $30 \mathrm{sec}$ ). $1 \mu \mathrm{l}$ of $1: 1$ mixture of ConA-functionalized GNRs and MCO-IImCherry plasmids was injected intravitreally into $\mathrm{rd10}$ mice eye to result in final concentration of 500 $\mathrm{ng} / \mathrm{ml}$ and $30 \mathrm{ng} / \mathrm{ml}$ respectively for GNRs and MCO-II-mCherry plasmids. After dilation, the CW NIR laser beam was applied for 30 seconds on the eye (Fig. 4b). Fig. 4c shows the zoomed image of an eye during the NOD laser exposure. The image of fundus with the NOD laser irradiation spot (red) is shown in Fig. 4d. 7 days after intravitreal injection and NOD, the mice were sacrificed, and retinal cup extracted and imaged using confocal fluorescence microscopy. While the non-targeted regions did not show reporter (mCherry) fluorescence (Fig. 4e), significant expression of reporter fluorescence in NODtargeted retinal area(s) was observed (Figs. 4 f, g). Comparison of fluorescence intensity of mCherry in targeted and non-targeted regions of retina is shown in Fig. 4h. The retina of non-NOD control eye did not show any characteristic (mCherry) fluorescence. Suppl. Fig. 2 shows cellular expression of mCherry in retina extracted after in-vivo NOD of MCO-II-mCherry. Suppl. Fig. 2b shows the intensity profiles along lines drawn through retinal cells (in Supp. Fig. 2a) expressing MCO-II-mCherry after NOD. Higher intensity of mCherry fluorescence in membrane demonstrated preferred localization of MCO-II in the membrane of retinal cells as compared to cytoplasm. During intravitreal injection of MCO-IImCherry plasmids and gold nanorods, there may be an injection related retinal damage (such as retinal detachment). Therefore, we evaluated structural integrity of retinal tissue and other ocular elements (cornea, lens) after injection (Suppl. Fig. 3 a, b). In order to characterize any damage due to NOD laser beam irradiation, the cornea and retina was imaged after NOD (Suppl. Fig. 3 c, d). Comparison of retinal thickness (No injection vs. After injection before NOD vs. After NOD) shows integrity of the retina is not compromised after NOD (Suppl. Fig. 3e).

\subsection{MCO-II sensitized RGCs after NOD are functionally activatable by white-light}

Functional response of the MCO-II expressing RGCs (in explant, delivered by NOD) toward whitelight stimulation was determined by multi-electrode array electrophysiology (Fig. 5a). Different light intensities were used to stimulate MCO-II expressing RGCs. Fig. 5b shows representative spiking activities in MCO-II transfected RGCs (after NOD) with and without light. With increase in light intensity the spiking rate increased (Fig. 5c). 


\section{Discussion}

Patient-to-patient variability and time-dependent changes in spatial-distribution of retinaldegeneration demands site-specific molecular delivery for effective therapeutic outcome. For example, in case of dry-AMD, spatially targeted delivery of opsin-encoding gene is required in macula, which loses photosensitivity due to loss of photoreceptors ${ }^{48.50}$. With viral or other non-viral (e.g. electroporation, lipofection) method, the opsin-genes will be delivered everywhere, causing uncontrolled expression over the whole retina. This will cause complications in functioning of non-degenerated areas of retina ${ }^{51}$. The spatially targeted delivery into regions of degenerated retina can be achieved using NOD by shining the NIR laser beam in targeted areas. The efficient localized field enhancement by gold nanostructures ${ }^{52 \cdot 60}$ in the safe NIR spectrum allowed in vitro and in vivo optical delivery of MCO-II-mCherry plasmid into the mammalian cells, retinal explant and targeted retinal regions of $r d 10$ mice. The significantly reduced white light stimulation intensity required for activating MCO-II sensitized cells will lead to ambient white light-based restoration of vision in case of retinal degenerative diseases. The NOD technology is easy to adapt in clinical setting since it puts no restriction on the maintaining the focal plane with target layer unlike focused ultrafast laser based optoporation. Further, it provides increased sensitivity of targeted cells with no detectable collateral damage. In addition, nano-enhancement near gold nanorods (used in NOD) allows use of low power, compact, less expensive and easy-to-use lasers.

Further, in case of loss of opsin expression in retina or development of new atrophies, it will be necessary to re-induce the opsin-encoding genes. The viral vectors have limitations in subsequent gene delivery in humans due to immunorejection and are unable to substantially re-induce transgene expression by reinjection in rodents ${ }^{6}$. In contrast, NOD has the ability for reinduction of genes to retina, providing an important advantage over viral vectors. Furthermore, the current state-of-the-art method for preclinical and clinical ocular gene therapy uses viral vectors, especially AAV vectors, which have selective tropism for certain retinal cell types. There often is a need to target multiple retinal cell types. For example, in glaucoma and ischemic retinopathy one need to target RGCs, microglia, astrocytes, and invading macrophages ${ }^{26-63}$. By varying shape of functionalized field-enhancing gold nanorods that binds to targeted cell types, tuning the NIR laser wavelength will allow wavelength-selective enhancement near specific cells and thus, selective delivery.

In NOD method, the contrast in temperature rise in laser-irradiated nanorod-attached cells at nanohotspots is significant enough to allow site-specific delivery of large (MCO-II-mCherry) plasmids. The NOD approach based on CW NIR laser beam is found to be minimally invasive with no detectable col- 
lateral damage to retina or other ocular elements as measured by OCT. Further, the gold nanorods have very low cytotoxicity and have been used in clinical trials. In case of rapid movement of eye in clinical practice, it may be advantageous to use a spatially sculpted NIR beam to match the shape of the region(s) of interest in the tissue requiring targeted molecular delivery by NOD. Shaping the NIR laser beam for NOD can be achieved by use of digital micromirror device (DMD) or spatial light modulator (SLM) to fit the targeted regions (e.g., geographic atrophies of retina in degenerative diseases) so that the therapeutic molecules (e.g. genes) can be delivered in a high throughput manner.

\section{Conclusions}

The in-vitro and in-vivo results, presented here using NOD, clearly demonstrate in-vivo gene delivery and functional cellular expression in targeted regions of retina using CW NIR laser beam without compromising structural integrity of the eye. Our results show that significant photocurrent can be reliably generated in MCO-II sensitized cells at white light intensity level close to ambient light condition. We believe our method of non-viral delivery of gene encoding opsins will pave the way for clinical applications in treatment of patients with retinal dystrophies.

\section{Author Contributions}

The manuscript was written through contributions of all authors. / All authors have given approval to the final version of the manuscript.

\section{Funding Sources}

National Institute of Health (1R43EY025905-01, 1R43EY026483-01, 3R43EY025905-01S1, 1R01EY025717-01A1)

\section{Conflict of Interest Statement}

The authors Sulagna Bhattacharya and Samarendra Mohanty have equity interest in Nanoscope Technologies LLC.

\section{Acknowledgement}

The authors would like to thank Dr. Sanjay Pradhan (Nanoscope) for animal care and Harvey Wiggins (Plexon Inc) for MEA equipment. 


\section{REFERENCES}

1. Hauswirth, W. W.; Aleman, T. S.; Kaushal, S.; Cideciyan, A. V.; Schwartz, S. B.; Wang, L., et al., Treatment of leber congenital amaurosis due to RPE65 mutations by ocular subretinal injection of adeno-associated virus gene vector: short-term results of a phase I trial. Hum.Gene Ther. 2008, 19, 979990.

2. Ng, E. W.; Shima, D. T.; Calias, P.; Cunningham, E. T.; Guyer, D. R.; Adamis, A. P., Pegaptanib, a targeted anti-VEGF aptamer for ocular vascular disease. Nature Reviews Drug Discovery 2006, 5, 123-132.

3. Burnett, J. C.; Rossi, J. J., RNA-based therapeutics: current progress and future prospects. Chemistry \& Biology 2012, 19, 60-71.

4. Herz, J.; Gerard, R. D., Adenovirus-mediated transfer of low density lipoprotein receptor gene acutely accelerates cholesterol clearance in normal mice. Proc.Natl.Acad.Sci U.S.A 1993, 90, 28122816.

5. Simon, R. H.; Engelhardt, J. F.; Yang, Y.; Zepeda, M.; Weber-Pendleton, S.; Grossman, M., et al., Adenovirus-mediated transfer of the CFTR gene to lung of nonhuman primates: toxicity study. Hum.Gene Ther. 1993, 4, 771-780.

6. Ali, M.; Lemoine, N. R.; Ring, C. J., The use of DNA viruses as vectors for gene therapy. Gene Ther. 1994, 1, 367-384.

7. Naldini, L.; Blömer, U.; Gallay, P.; Ory, D.; Mulligan, R.; Gage, F. H., et al., In Vivo Gene Delivery and Stable Transduction of Nondividing Cells by a Lentiviral Vector. Science 1996, 272, 263267.

8. Wu, Z.; Yang, H.; Colosi, P., Effect of genome size on AAV vector packaging. Mol.Ther. 2010, $18,80-86$.

9. Grunwald, J. E.; Pistilli, M.; Ying, G. S.; Maguire, M. G.; Daniel, E.; Martin, D. F., Growth of Geographic Atrophy in the Comparison of Age-related Macular Degeneration Treatments Trials. Ophthalmology 2015, 122, 809-816.

10. Wu, Z.; Ayton, L. N.; Luu, C. D.; Guymer, R. H., Microperimetry of nascent geographic atrophy in age-related macular degeneration. Invest Ophthalmol Vis Sci 2015, 56, 115-21.

11. Wallsh, J.; Gallemore, R., Optical coherence tomography difference maps and average macular volume for geographic atrophy. Retin Cases Brief Rep 2015, 9, 88-91.

12. Biarnes, M.; Mones, J.; Alonso, J.; Arias, L., Update on geographic atrophy in age-related macular degeneration. Optom Vis Sci 2011, 88, 881-9. 
13. Sunness, J. S.; Margalit, E.; Srikumaran, D.; Applegate, C. A.; Tian, Y.; Perry, D., et al., The long-term natural history of geographic atrophy from age-related macular degeneration: enlargement of atrophy and implications for interventional clinical trials. Ophthalmology 2007, 114, 271-7.

14. Matsuda, T.; Cepko, C. L., Electroporation and RNA interference in the rodent retina in vivo and in vitro. Proc.Natl.Acad.Sci U.S.A 2004, 101, 16-22.

15. Mohanty, S. K.; Ficinski, M.; Wong, E. K.; Berns, M. W., Method and apparatus for optogenetic treatment of blindness including retinitis pigmentosa. Google Patents: 2015; Vol. US9089698B2.

16. Wells, D. J., Electroporation and ultrasound enhanced non-viral gene delivery in vitro and in vivo. Cell Biology and Toxicology 2010, 26, 21-28.

17. Bejjani, R. A.; BenEzra, D.; Cohen, H.; Rieger, J.; Andrieu, C.; Jeanny, J. C., et al., Nanoparticles for gene delivery to retinal pigment epithelial cells. Mol Vis 2005, 11, 124-32.

18. Han, Z.; Koirala, A.; Makkia, R.; Cooper, M. J.; Naash, M. I., Direct gene transfer with compacted DNA nanoparticles in retinal pigment epithelial cells: expression, repeat delivery and lack of toxicity. Nanomedicine (Lond) 2012, 7, 521-39.

19. Wang, Y.; Rajala, A.; Cao, B.; Ranjo-Bishop, M.; Agbaga, M. P.; Mao, C., et al., Cell-Specific Promoters Enable Lipid-Based Nanoparticles to Deliver Genes to Specific Cells of the Retina In Vivo. Theranostics 2016, 6, 1514-27.

20. Wang, Y.; Rajala, A.; Rajala, R. V., Lipid Nanoparticles for Ocular Gene Delivery. J Funct Biomater 2015, 6, 379-94.

21. Li, S.; Huang, L., Nonviral gene therapy: promises and challenges. Gene Ther 2000, 7, 31-34.

22. Dhakal, K.; Black, B.; Mohanty, S., Introduction of impermeable actin-staining molecules to mammalian cells by optoporation. Scientific Reports 2014, 4, 6553.

23. Dhakal, K.; Batabyal, S.; Wright, W.; Kim, Y.-T.; Mohanty, S., Optical delivery of multiple opsin-encoding genes leads to targeted expression and white-light activation. Light: Science and Applications 2015, 4, e352.

24. Gu, L.; Mohanty, S. K., Targeted microinjection into cells and retina using optoporation. Journal of Biomedical Optics 2011, 16, 128003.

25. Batabyal, S.; Kim, Y.-T.; Mohanty, S. K., Ultrafast laser-assisted spatially targeted optoporation into cortical axons and retinal cells in the eye. Journal of Biomedical Optics 2017, 22, 060504.

26. Bi, A. D.; Cui, J. J.; Ma, Y. P.; Olshevskaya, E.; Pu, M. L.; Dizhoor, A. M., et al., Ectopic expression of a microbial-type rhodopsin restores visual responses in mice with photoreceptor degeneration. Neuron 2006, 50, 23-33. 
27. Thyagarajan, S.; van Wyk, M.; Lehmann, K.; Lowel, S.; Feng, G.; Wassle, H., Visual Function in Mice with Photoreceptor Degeneration and Transgenic Expression of Channelrhodopsin 2 in Ganglion Cells. J. Neurosci. 2010, 30, 8745-8758.

28. Zhang, Y.; Ivanova, E.; Bi, A.; Pan, Z.-H., Ectopic Expression of Multiple Microbial Rhodopsins Restores ON and OFF Light Responses in Retinas with Photoreceptor Degeneration. $J$. Neurosci. 2009, 29, 9186-9196.

29. Tomita, H.; Sugano, E.; Isago, H.; Hiroi, T.; Wang, Z.; Ohta, E., et al., Channelrhodopsin-2 gene transduced into retinal ganglion cells restores functional vision in genetically blind rats. Experimental Eye Research 2010, 90, 429-436.

30. Tomita, H.; Sugano, E.; Fukazawa, Y.; Isago, H.; Sugiyama, Y.; Hiroi, T., et al., Visual Properties of Transgenic Rats Harboring the Channelrhodopsin-2 Gene Regulated by the Thy-1.2 Promoter. PLoS One 2009, 4, e7679.

31. Gu, L.; Shivalingaiah, S.; Ficinski, M.; Wong, E.; Mohanty, S., Non-viral delivery and optimized optogenetic stimulation of retinal ganglion cells led to behavioral restoration of vision. Nature Precedings 2012.

32. Lagali, P. S.; Balya, D.; Awatramani, G. B.; Munch, T. A.; Kim, D. S.; Busskamp, V., et al., Light-activated channels targeted to $\mathrm{ON}$ bipolar cells restore visual function in retinal degeneration. Nat. Neurosci. 2008, 11, 667-675.

33. Doroudchi, M. M.; Greenberg, K. P.; Liu, J.; Silka, K. A.; Boyden, E. S.; Lockridge, J. A., et al., Virally delivered Channelrhodopsin-2 Safely and Effectively Restores Visual Function in Multiple Mouse Models of Blindness. Mol Ther 2011, 19, 1220-1229.

34. Wright, W.; Gajjeraman, S.; Batabyal, S.; Pradhan, S.; Bhattacharya, S.; Mahapatra, V., et al., Restoring vision in mice with retinal degeneration using multicharacteristic opsin. Neurophotonics $2017,4,041505$.

35. Busskamp, V.; Duebel, J.; Balya, D.; Fradot, M.; Viney, T. J.; Siegert, S., et al., Genetic Reactivation of Cone Photoreceptors Restores Visual Responses in Retinitis Pigmentosa. Science 2010, $329,413-417$.

36. Bainbridge, J. W.; Smith, A. J.; Barker, S. S.; Robbie, S.; Henderson, R.; Balaggan, K., et al., Effect of gene therapy on visual function in Leber's congenital amaurosis. N Engl J Med 2008, 358, 2231-9.

37. Ivanova, E.; Roberts, R.; Bissig, D.; Pan, Z.-H.; Berkowitz, B. A., Retinal channelrhodopsin-2mediated activity in vivo evaluated with manganese-enhanced magnetic resonance imaging. $J$. Molecular. Vision. 2010, 16, 1059-1067. 
38. Bi, A.; Cui, J.; Ma, Y. P.; Olshevskaya, E.; Pu, M.; Dizhoor, A. M., et al., Ectopic expression of a microbial-type rhodopsin restores visual responses in mice with photoreceptor degeneration. Neuron 2006, 50, 23-33.

39. Adler, D. C.; Huber, R.; Fujimoto, J. G., Phase-sensitive optical coherence tomography at up to 370,000 lines per second using buffered Fourier domain mode-locked lasers. Opt. Lett. 2007, 32, 626628.

40. Choma, M. A.; Ellerbee, A. K.; Yang, C.; Creazzo, T. L.; Izatt, J. A., Spectral-domain phase microscopy. Opt. Lett. 2005, 30, 1162-1164.

41. Joo, C.; Akkin, T.; Cense, B.; Park, B. H.; de Boer, J. F., Spectral-domain optical coherence phase microscopy for quantitative phase-contrast imaging. Opt. Lett. 2005, 30, 2131-2133.

42. Joo, C.; Kim, K. H.; de Boer, J. F., Spectral-domain optical coherence phase and multiphoton microscopy. Opt. Lett. 2007, 32, 623-625.

43. Sarunic, M. V.; Weinberg, S.; Izatt, J. A., Full-field swept-source phase microscopy. Opt. Lett. $2006,31,1462-1464$.

44. Wang, R. K.; Nuttall, A. L., Phase-sensitive optical coherence tomography imaging of the tissue motion within the organ of Corti at a subnanometer scale: a preliminary study. Journal of Biomedical Optics 2010, 15, 056005-9.

45. Liu, Y.; McDowell, C. M.; Zhang, Z.; Tebow, H. E.; Wordinger, R. J.; Clark, A. F., Monitoring retinal morphologic and functional changes in mice following optic nerve crush. Invest Ophthalmol Vis Sci 2014, 55, 3766-74.

46. Kim, B. J.; Silverman, S. M.; Liu, Y.; Wordinger, R. J.; Pang, I. H.; Clark, A. F., In vitro and in vivo neuroprotective effects of cJun N-terminal kinase inhibitors on retinal ganglion cells. Mol Neurodegener 2016, 11, 30 .

47. Kim, B. J.; Sprehe, N.; Morganti, A.; Wordinger, R. J.; Clark, A. F., The effect of postmortem time on the RNA quality of human ocular tissues. Mol Vis 2013, 19, 1290-5.

48. Klein, M. L.; Ferris, F. L., 3rd; Francis, P. J.; Lindblad, A. S.; Chew, E. Y.; Hamon, S. C., et al., Progression of geographic atrophy and genotype in age-related macular degeneration. Ophthalmology 2010, 117, 1554-1559.

49. Fleckenstein, M.; Schmitz-Valckenberg, S.; Adrion, C.; Kramer, I.; Eter, N.; Helb, H. M., et al., Tracking progression with spectral-domain optical coherence tomography in geographic atrophy caused by age-related macular degeneration. Invest Ophthalmol Vis Sci 2010, 51, 3846-52. 
50. Sunness, J. S.; Applegate, C. A.; Bressler, N. M.; Hawkins, B. S., Designing clinical trials for age-related geographic atrophy of the macula: enrollment data from the geographic atrophy natural history study. Retina 2007, 27, 204-10.

51. Jacobson, S. G.; Roman, A. J.; Aleman, T. S.; Sumaroka, A.; Herrera, W.; Windsor, E. A., et al., Normal central retinal function and structure preserved in retinitis pigmentosa. Invest Ophthalmol Vis Sci 2010, 51, 1079-85.

52. Wang, S.; Chen, K. J.; Wu, T. H.; Wang, H.; Lin, W. Y.; Ohashi, M., et al., Photothermal Effects of Supramolecularly Assembled Gold Nanoparticles for the Targeted Treatment of Cancer Cells. Angewandte Chemie 2010, 122, 3865-3869.

53. Cheng, Y.; Samia, A. C.; Li, J.; Kenney, M. E.; Resnick, A.; Burda, C., Delivery and efficacy of a cancer drug as a function of the bond to the gold nanoparticle surface. Langmuir 2010, 26, 2248-55.

54. Kim, B.; Han, G.; Toley, B. J.; Kim, C.-k.; Rotello, V. M.; Forbes, N. S., Tuning payload delivery in tumour cylindroids using gold nanoparticles. Nat Nano 2010, 5, 465-472.

55. Tong, L.; Zhao, Y.; Huff, T.; Hansen, M.; Wei, A.; Cheng, J. X., Gold Nanorods Mediate Tumor Cell Death by Compromising Membrane Integrity. Advanced Materials 2007, 19, 3136-3141.

56. Huang, X.; El-Sayed, I. H.; Qian, W.; El-Sayed, M. A., Cancer Cell Imaging and Photothermal Therapy in the Near-Infrared Region by Using Gold Nanorods. Journal of the American Chemical Society 2006, 128, 2115-2120.

57. Gobin, A. M.; Lee, M. H.; Halas, N. J.; James, W. D.; Drezek, R. A.; West, J. L., Near-Infrared Resonant Nanoshells for Combined Optical Imaging and Photothermal Cancer Therapy. Nano Letters 2007, 7, 1929-1934.

58. Brongersma, M. L., Nanoscale photonics: Nanoshells: gifts in a gold wrapper. Nat Mater 2003, 2, 296-297.

59. Chen, J.; Wang, D.; Xi, J.; Au, L.; Siekkinen, A.; Warsen, A., et al., Immuno Gold Nanocages with Tailored Optical Properties for Targeted Photothermal Destruction of Cancer Cells. Nano Letters $2007,7,1318-1322$.

60. Chen, J.; Saeki, F.; Wiley, B. J.; Cang, H.; Cobb, M. J.; Li, Z.-Y., et al., Gold Nanocages:

Bioconjugation and Their Potential Use as Optical Imaging Contrast Agents. Nano Letters 2005, 5, 473477.

61. Mori, K.; Gehlbach, P.; Ando, A.; Wahlin, K.; Gunther, V.; McVey, D., et al., Intraocular Adenoviral Vector-Mediated Gene Transfer in Proliferative Retinopathies. Investig. Ophthalmol. Vis. Sci. 2002, 43, 1610-1615. 
62. Howell, G. R.; Macalinao, D. G.; Sousa, G. L.; Walden, M.; Soto, I.; Kneeland, S. C., et al., Molecular clustering identifies complement and endothelin induction as early events in a mouse model of glaucoma. J Clin Invest 2011, 121, 1429-44.

63. Silverman, S. M.; Kim, B. J.; Howell, G. R.; Miller, J.; John, S. W.; Wordinger, R. J., et al., C1q propagates microglial activation and neurodegeneration in the visual axis following retinal ischemia/reperfusion injury. Mol Neurodegener 2016, 11, 24. 


\section{Figures and captions:}

a

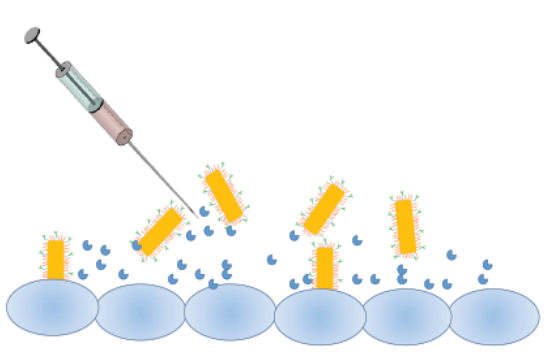

Add molecules to-be-delivered into the medium

b

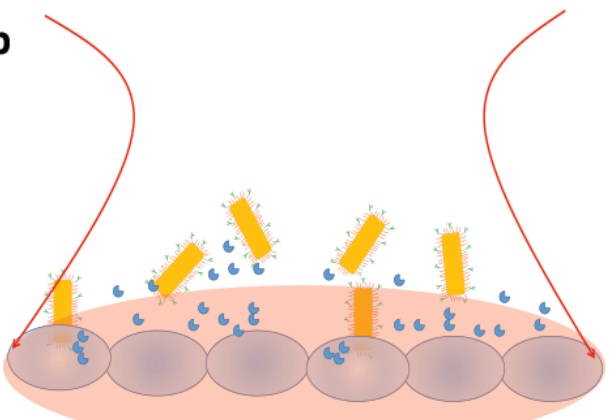

Molecules enter via membrane exposed to hotspots

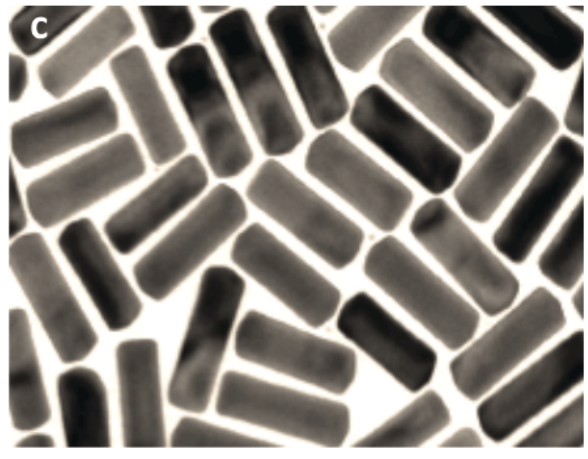

d

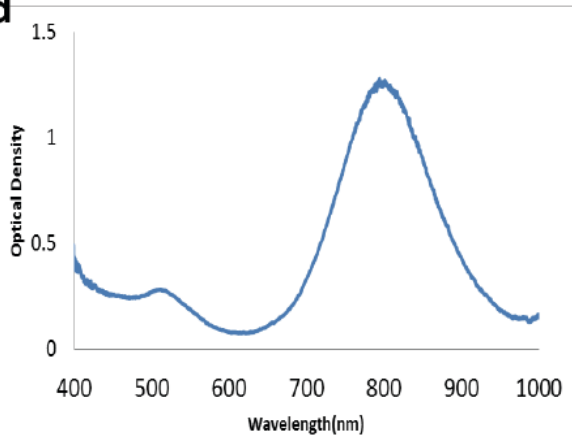

Fig 1. Principle of Nano-enhanced Optical Delivery (NOD). (a) The gold nanorods (injected intravitreally) bind to targeted retinal cells. (b) Upon illumination of a continuous wave laser of specific wavelength, the rods generate localized hot spots on membrane, allowing insertion of genes into the targeted (geographic atrophy) area(s) of retina. (c) Electronic microscopic image of GNRs. For binding of GNRs to cells. 

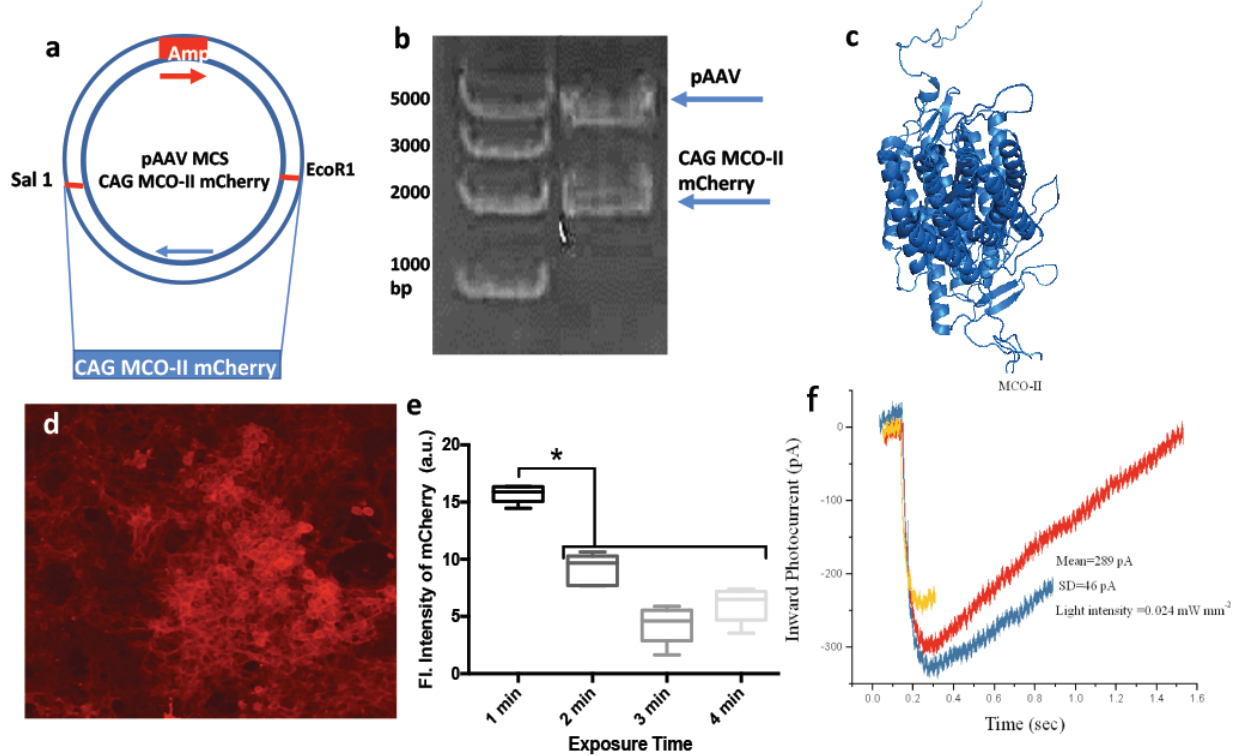

Fig 2. NOD optimized for delivery of Multi-Characteristic Opsin (MCO-II). (a) A typical circular map showing the insertion of MCO-II gene cloned at the restriction sites (Sal1 and EcoR1); (b) Agarose gel electrophoresis of CAG-MCO-II-mCherry gene (2915 bp) after restriction enzyme digestion with EcoR1 and Sal1; (c) Theoretical modeling of the three-dimensional arrangement of amino acid chains of MCO-II. (d) Confocal fluorescence image showing expression of MCO-II-mCherry in the HEK cells after NOD (6.4 $\mathrm{mW} / \mathrm{mm}^{2}$ and $1 \mathrm{~min}$ exposure); (e) Intrinsic fluorescence intensity of mCherry with different laser beam $(800 \mathrm{~nm})$ exposure time, ${ }^{*} \mathrm{p}<0.01$ between $1 \mathrm{~min}$ and others. (f) Inward current in MCO-II expressing cells in response to light (average intensity: $0.024 \mathrm{~mW} / \mathrm{mm}^{2}$ ) measured by Patchclamp electrophysiology. 

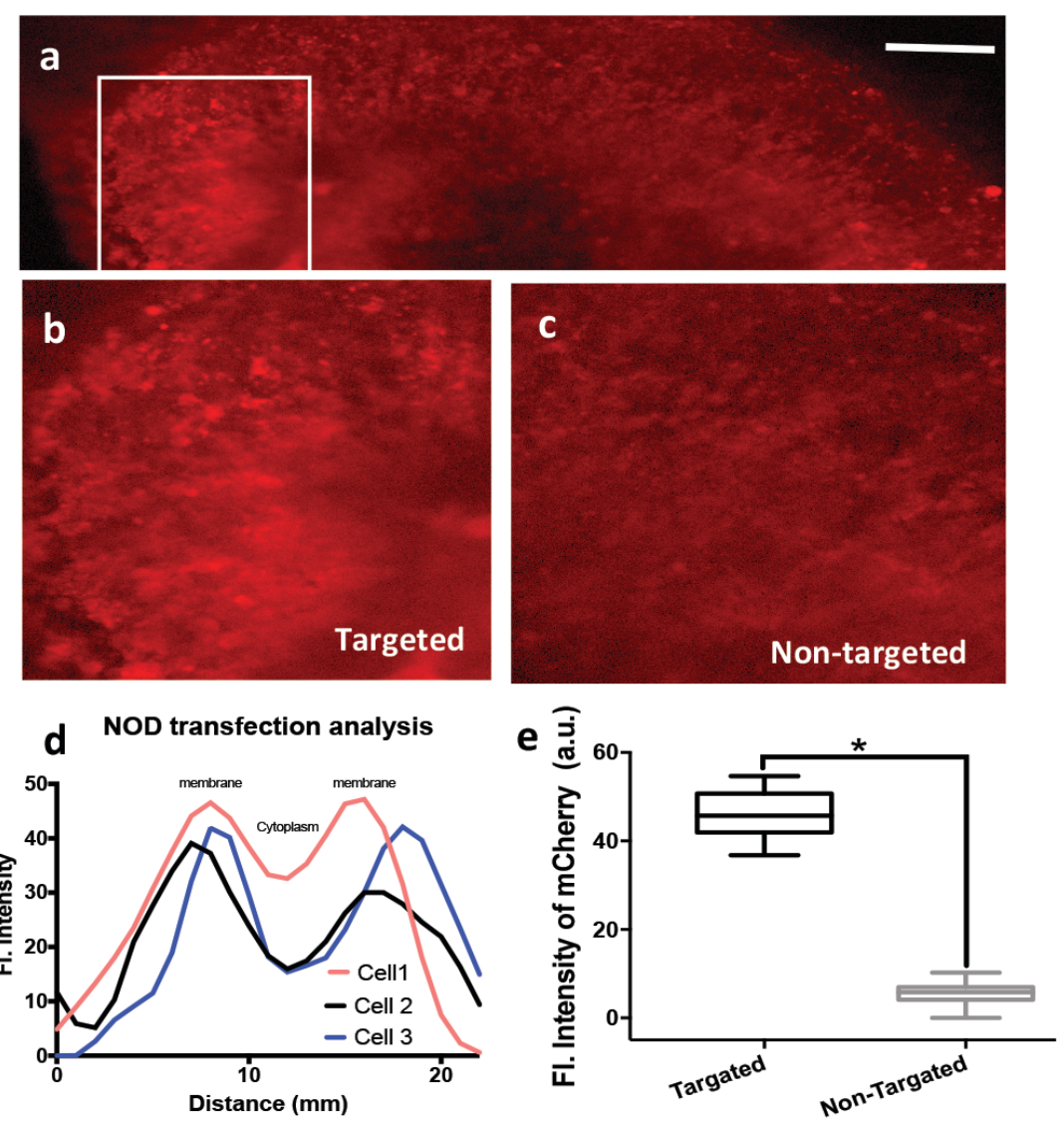

Fig 3. Nano-enhanced Optical delivery (NOD) of MCO-II-mCherry plasmids to retinal explants.

(a) Confocal fluorescence image of retinal explant showing the expression of MCO-II in targeted area (marked by square) 2 days after exposure of CW laser (30 sec) in presence of GNR (800nm) and MCOII plasmids, (b) Zoomed image of targeted area expressing mCherry, and (c) zoomed image of nontargeted area in an explant. (d) Intensity profile along lines drawn through the retinal cells showing preferential expression of MCO-II-mCherry after NOD in the cell membrane; (e) Comparison of MCOII-mCherry expression in targeted versus non-targeted regions in retina explant. 


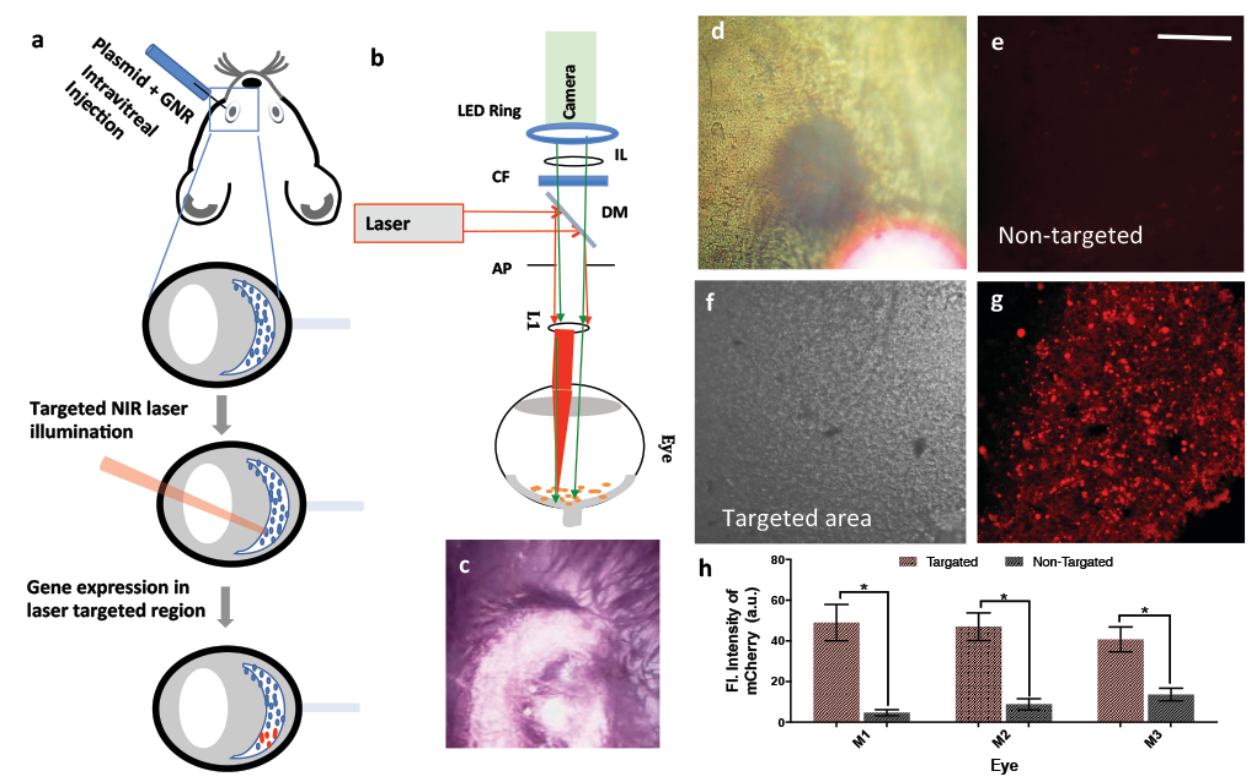

Fig 4. In-vivo targeted Nano-enhanced Optical delivery (NOD) of MCO-II-mCherry plasmids to retina. (a) Experimental scheme for in-vivo NOD in eye; (b) Schematic of experimental setup for invivo NOD. (c) Picture showing mouse eye during in-vivo NOD of the eye, injected with GNR and MCO-II plasmids; (d) In-vivo fundus image of retina during laser exposure. (e) Confocal fluorescence image of non-targeted region 7 days after NOD; (f) Bright field confocal image of the targeted region; (g) confocal fluorescence image of showing MCO-II mCherry expression 7 days after NOD; (h) Comparison of fluorescence intensity of mCherry in targeted and non-targeted regions. ${ }^{*} \mathrm{p}<0.01$ between targeted and non-targeted; $\mathrm{N}=10$ cells/ mouse. 

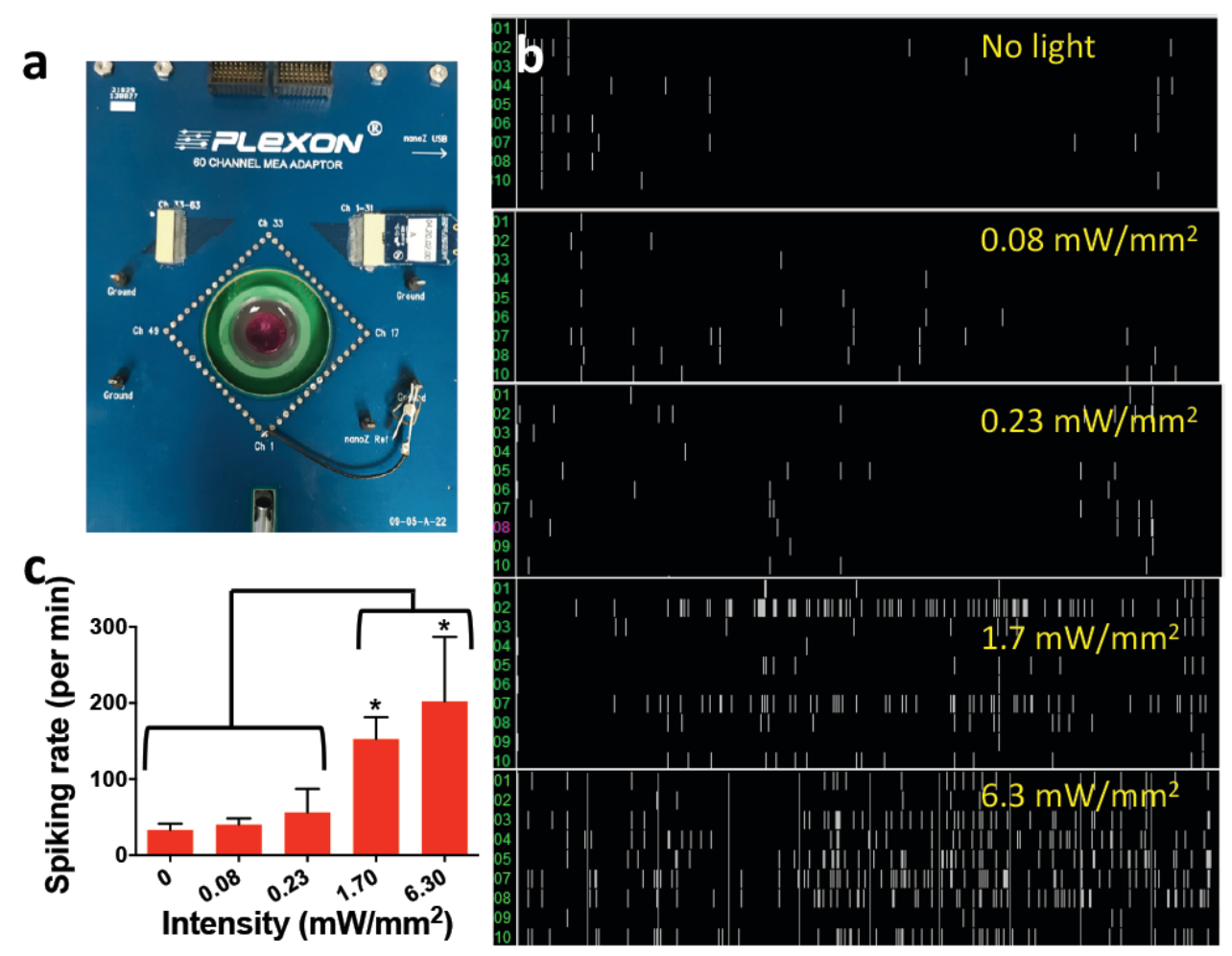

Fig. 5. White light-activated spiking activities in MCO-II transfected RGCs in retina explant after NOD. (a) Multi electrode array chip to measure light-activated spiking in retina after MCO-II transfection using NOD. (b) White light evoked spiking activities in retina transfected with MCO-II by NOD. $1 \mathrm{~Hz}$ light pulses (200 ms ON, $800 \mathrm{~ms}$ OFF) for $60 \mathrm{sec}$. (c) Stimulation light intensity dependent spiking in retina transfected with MCO-II by NOD. $* \mathrm{p}<0.05$. 


\title{
Supplemental Information
}

\section{Nano-enhanced optical gene delivery to retinal degenerated mice}

\author{
Subrata Batabyal; Sivakumar Gajjeraman", Sulagna Bhattacharya, Weldon Wright, Samarendra Mohanty*
}

Nanoscope Technologies LLC, 1312 Brown Trail

Bedford, Texas, USA, 76022

†These authors contributed equally.

Corresponding Author

* E-mail: smohanty@nanoscopetech.com 


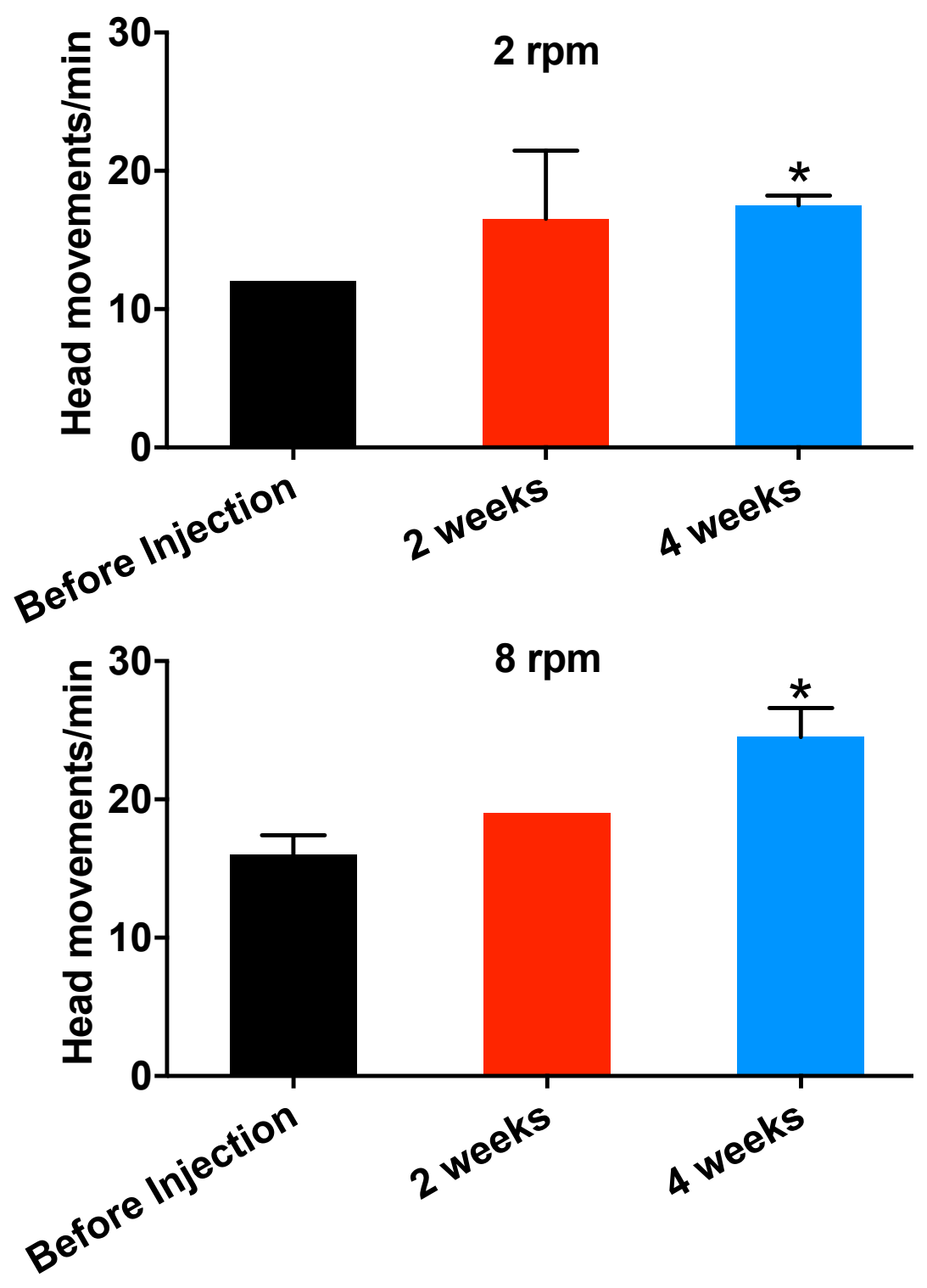

Suppl. Fig. 1. Improvement of optokinetic response in MCO-II transfected rd10 mice at ambient light level. Quantitative comparison of number of head movement at different speed of rotation of the vertical stripes: (Top) $2 \mathrm{rpm}$ and (Bottom) $8 \mathrm{rpm}$ before and after MCO-II transfection. $r d 10$ mice with MCO-II transfection shows improved optokinetic response as reflected in the increase head movement. The average light intensity at the center of the chamber was $0.001 \mathrm{~mW} / \mathrm{mm}^{2}$. 


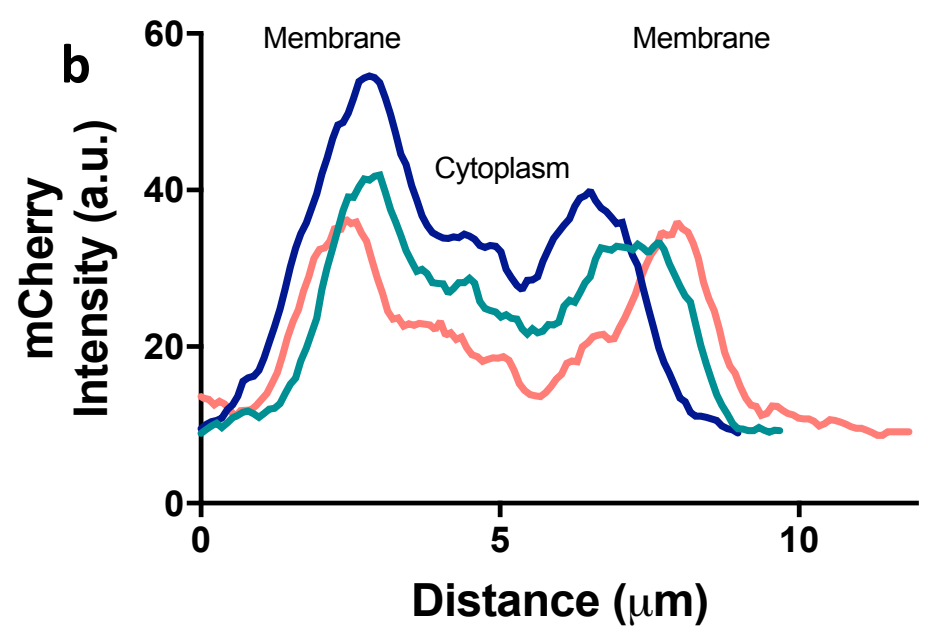

Suppl. Fig. 2. (a) Confocal image showing expression of MCO-II in the retinal cells after $\sim 1$ wk of NOD. (b) Intensity profile along lines drawn through the cells expressing MCO-II-mCherry after NOD. 

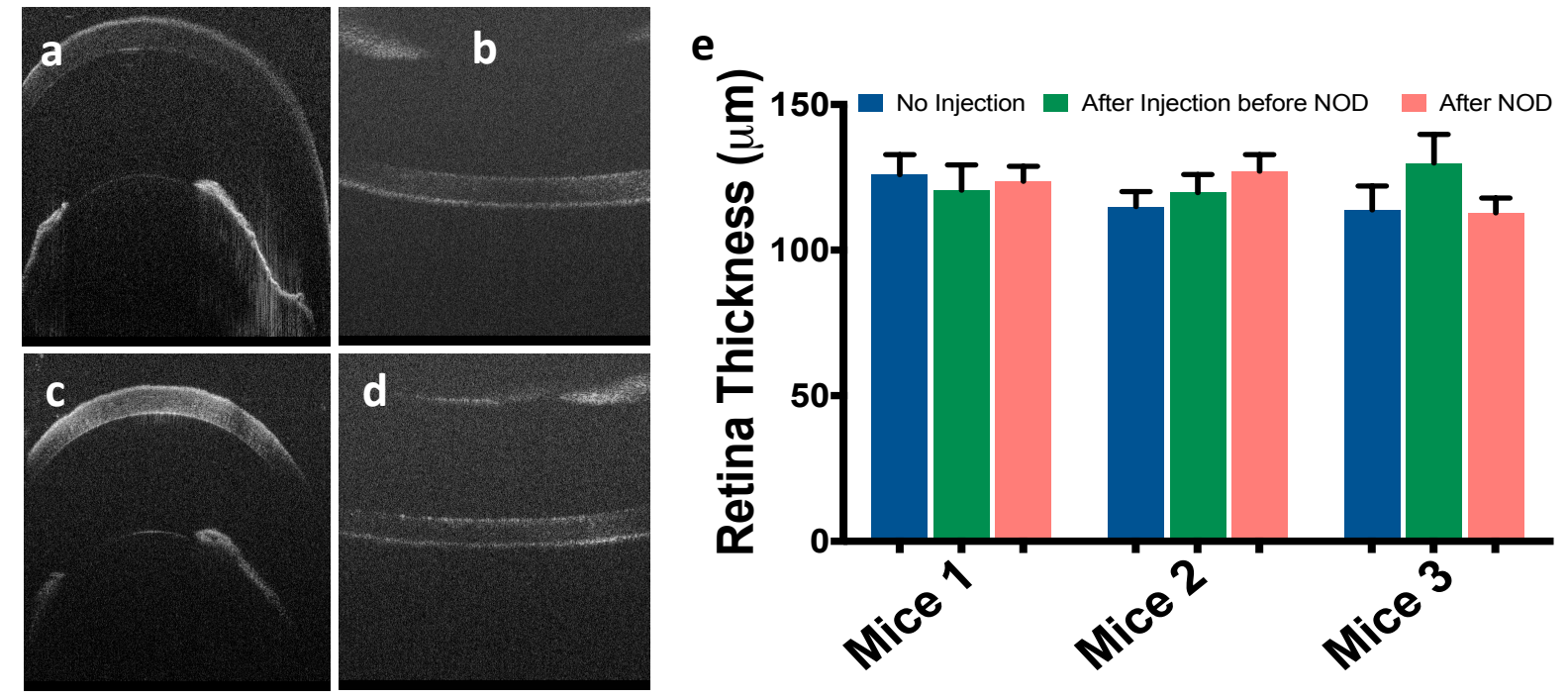

Suppl. Fig. 3. (a) Monitoring changes in cornea and retina using SDOCT after NOD. OCT image of (a) cornea and lens (and iris) and (b) retina before optical delivery. OCT image of (c) cornea and lens (and iris) and (d) retina of same animal after optical gene delivery to retina. (e) Comparison of retinal thickness (No injection vs. After injection before NOD vs After NOD). 